\title{
Effect of Cheonggukjang supplementation upon hepatic acyl-CoA synthase, carnitine palmitoyltransferase I, acyl-CoA oxidase and uncoupling protein 2 mRNA levels in C57BL/6J mice fed with high fat diet
}

\author{
Ju-Ryoun Soh · Dong-Hwa Shin · Dae Young Kwon · \\ Youn-Soo Cha
}

Published online: 15 November 2007

(C) Springer-Verlag 2007

\begin{abstract}
This study investigated the effect of Cheonggukjang on mRNA levels of hepatic acyl-CoA synthase (ACS), carnitine palmitoyltransferase I (CPT-I), acyl-CoA oxidase (ACO) and uncoupling protein 2 (UCP2), and on serum lipid profiles in C57BL/6J mice. Thirty male C57BL/ $6 \mathrm{~J}$ mice were divided into three groups; normal diet (ND), high fat diet (HD) and high fat diet with $40 \%$ Cheonggukjang (HDC). Energy intake was significantly higher in the HDC group than in the ND and HD groups. The HDC group normalized in weight gain, epididymal and back fat $(\mathrm{g} /$ $100 \mathrm{~g}$ ) accumulation which are increased by high fat diet. Serum concentrations of triglyceride and total cholesterol in the HDC were significantly lower than those in the HD group. These results were confirmed by hepatic mRNA expression of enzymes and protein (ACS, CPT-1, ACO, UCP2) which is related with lipid metabolism by RT-PCR. Hepatic CPT-I, ACO and UCP2 mRNA expression was increased by Cheonggukjang supplementation. We demonstrated that Cheonggukjang supplement leads to increased mRNA expressions of enzymes and protein involved in fatty acid oxidation in liver, reduced accumulation of body fat and improvement of serum lipids in high fat diet fed mice.
\end{abstract}

J.-R. Soh · Y.-S. Cha ( $\bowtie)$

Department of Food Science and Human Nutrition, Research Institute of Human Ecology, Chonbuk National University, 664-14 Dukjin-Dong 1-Ga, Jeonju 561-756, Republic of Korea e-mail: cha8@chonbuk.ac.kr

\section{D.-H. Shin}

Department of Food Science and Technology, Chonbuk National University, Jeonju 561-756, Republic of Korea

D. Y. Kwon

Division of Food Functional Research, Korean Food Research Institutes, Songnam, Kyongki-do 463-746, Republic of Korea
Keywords Carnitine palmitoyl transferase I (CPT-I) . Fermented soybean · High fat diet · Lipid metabolism . Uncoupling protein 2 (UCP 2)

\section{Introduction}

Formation of fatty acyl coenzyme A (CoA) from fatty acid, adenosine triphosphate (ATP), and CoA catalyzed by acylCoA synthetase (ACS) is the first reaction in fatty acid metabolism. Acyl-CoA synthase plays a critical role in lipid synthesis of triacylglycerols (TGs), phospholipids, cholesterol esters, and $\beta$-oxidation of fatty acid. Acyl-CoA synthase, a member of the luciferase gene family [20], is located on microsomes, outer mitochondrial membranes, and peroxisomes in rat liver [11]. Carnitine palmitoyltransferase-I (CPT-I) is the rate-limiting enzyme for fatty acid oxidation. This enzyme catalyzes the formation of acyl-carnitine from acyl-CoA, the first step in the transport of long-chain fatty acids from the cytosol into the mitochondrial matrix for fatty acid oxidation [20, 23]. Uncoupling proteins (UCPs) are mitochondrial inner membrane proteins. Uncoupling proteins allow the dissipation of part of the proteon electrochemical gradient generated by the electron transfer chain across the mitochondrial inner membrane and can thus increase heat production by uncoupling respiration from ATP synthesis [1]. Uncoupling protein 2 (UCP2) mRNA is expressed in numerous types of tissue such as liver, skeletal muscle, heart, and kidney. UCP3 is expressed mainly in the skeletal muscle [16]. Acyl-CoA oxidase catalyzes the first step of the $\beta$-oxidation cycle in which a fatty acyl-CoA is oxidized to 2-trans-enoyl-CoA. During this step, the FAD-containing acyl-CoA oxidase donates electrons directly to molecular oxygen resulting in the formation of hydrogen 
peroxide. Genes encoding acyl-CoA oxidase have been isolated from rat [12].

Soybean-based fermented foods such as Cheonggukjang are very popular in Korea. Cheonggukjang has traditionally been made with whole cooked soybean by fermenting with Bacillus subtilis for short-periods (2 days) without salt addition. During fermentation, isoflavonoids are converted from glycosides into the corresponding aglycones, and most proteins are degraded into small peptides and amino acids [15, 19]. Cheonggukjang is known to have a fibrinolytic enzyme that may have a role in removing blood clots [8]. Cheonggukjang also exhibits anti-cancer properties, reduces blood pressure, and has hypocholesterolemic effects in serum [25]. However, there have been few studies on the beneficial effects of Cheonggukjang on $\beta$-oxidation of fatty acids in high fat diet induced obesity.

In this study, we investigated the effects of Cheonggukjang supplementation on weight gain, epididymal and back fat accumulation, lipid profiles, and hepatic mRNA expression of enzymes and proteins (ACS, CPT-1, ACO, UCP2) related to lipid metabolism in high fat diet induced obesity.

\section{Materials and methods}

Experimental animal and diets

Cheonggukjang was prepared by the traditional processing method at the Department Food Science and Technology of Chonbuk National University (Jeonju, Korea). Male 4week-old C57BL/6J mice were fed a standard commercial diet for 1 week and then divided into three groups by randomized block design. During the experimental periods, the animals were given free access to water and the modified AIN-93 diets: normal diet control group (ND), high fat diet control group (HD), and high fat diet plus $40 \%$ Cheonggukjang (HDC). After being maintained on their respective diets for 12 weeks, animals were sacrificed after fasting for $12 \mathrm{~h}$. Dietary intake and weight were recorded every other day and weekly, respectively. Room temperature and humidity were maintained at $23 \pm 1{ }^{\circ} \mathrm{C}$ and $53 \pm 2 \%$, respectively, with a 12:12 h light/dark cycle. Fat content of the high fat diet was $24 \%$ ( $45 \%$ of total calories from fat), and fat content of the normal diet was $4 \%$ (10\% of total calories from fat (Research Diet, New Brunswick, N.J., USA).

Sample collection and lipid analysis

To reduce the effects of differences in dietary intake, blood samples were collected after $12 \mathrm{~h}$ overnight fasting by orbital venipuncture. Blood samples were left on ice for
$1 \mathrm{~h}$, centrifuged at $1,100 \mathrm{~g}$ within $15 \mathrm{~min}$, and separated serum collected. Serum samples were stored at $-80^{\circ} \mathrm{C}$ until analysis. After blood collection, organs such as liver, epididymal fat, and back fat were surgically removed, washed in saline to remove foreign substances, dried on filter paper and stored in labeled 1.5-ml Eppendorf tubes. Serum and liver lipid levels were estimated by an enzymatic colorimetric method using a commercial assay kit (Asan Pharm, Seoul, Korea).

mRNA levels in liver

Total RNA from the livers were isolated using the TRI reagent (Molecular Research, Cincinnati, Ohio, USA) according to the manufacturer's instructions. Total RNA was quantified by measuring absorption at 260 and $280 \mathrm{~nm}$. ACS, CPT-I, ACO and UCP2 mRNA expression levels were measured through RT-PCR (the reverse transcriptase-PCR method). The RT-PCR was conducted using $1 \mu \mathrm{g}$ denaturated RNA and random hexamer primers as described by the manufacturer (one-step RT-PCR kit from ABgene, NY). The cDNA product was amplified in a 50- $\mu 1$ final volume containing each of the primers (of ACS, CPTI, ACO and UCP2) and RT-PCR Master Mix (thermoprime plus DNA polymerase, and an optimized reaction buffer, dNTP mix and $\mathrm{MgCl}_{2}$ ).

\section{Statistical analysis}

Significance of the differences between the samples was determined by one-way analysis of variance (ANOVA) using SAS software version 8 (SAS Institute, USA). Differences within the three groups were separated using Duncan's multiple range test. The accepted level of significance was $P<0.05$. Results were expressed as mean $\pm \mathrm{SD}$.

\section{Results}

We measured the body weight of 4-week-old mice $(20.0 \pm 0.2 \mathrm{~g})$ just before starting the feeding programs and randomized each into three different groups: ND, HD and HDC. As depicted in Table 1, energy intake was significantly higher in the HDC group compared with the ND group and the HD group. Twelve weeks feeding of C57BL/ $6 \mathrm{~J}$ mice with the HD resulted in significant increases in body weight $(120 \%, P<0.05)$, epididymal fat $(200 \%$, $P<0.05)$ and back fat $(140 \%, P<0.05)$, as compared to the ND-fed mice, but was normalized in the HDC group. Plasma triglyceride and total cholesterol levels were 1.42- 
Table 1 Weight-related and biochemical parameters in mice

Values are means $\pm \mathrm{SD}$

$N D$ Normal diet control, $H D$ high fat diet control, $H D C$ HD plus $40 \%$ Cheonggukjang

Values with different letters within the same row are significantly different at $P<0.05$ by Duncan's multiple range test

\begin{tabular}{llrr}
\hline & ND & HD & \multicolumn{1}{l}{ HDC } \\
\hline Energy intake $(\mathrm{kcal} / \mathrm{day})$ & $6.50 \pm 0.16 \mathrm{~b}$ & $6.53 \pm 0.03 \mathrm{~b}$ & $7.01 \pm 0.24 \mathrm{a}$ \\
Weight gain $(\mathrm{g})$ & $6.98 \pm 0.91 \mathrm{~b}$ & $8.60 \pm 1.57 \mathrm{a}$ & $6.54 \pm 1.42 \mathrm{~b}$ \\
Epididymal fat $(\mathrm{g} / 100 \mathrm{~g} \mathrm{bw})$ & $1.73 \pm 0.31 \mathrm{c}$ & $3.52 \pm 0.53 \mathrm{a}$ & $2.60 \pm 0.81 \mathrm{~b}$ \\
Back fat $(\mathrm{g} / 100 \mathrm{~g} \mathrm{bw})$ & $2.00 \pm 0.41 \mathrm{~b}$ & $2.83 \pm 0.82 \mathrm{a}$ & $2.25 \pm 0.72 \mathrm{~b}$ \\
Serum & & & \\
Triglyceride $(\mathrm{mg} / \mathrm{dl})$ & $100.42 \pm 27.64 \mathrm{~b}$ & $142.13 \pm 21.05 \mathrm{a}$ & $93.94 \pm 13.83 \mathrm{~b}$ \\
Total cholesterol $(\mathrm{mg} / \mathrm{dl})$ & $170.67 \pm 55.95 \mathrm{~b}$ & $292.96 \pm 35.32 \mathrm{a}$ & $182.77 \pm 24.61 \mathrm{~b}$ \\
Liver & & & \\
Triglyceride $(\mathrm{mg} / \mathrm{g})$ & $3.05 \pm 0.91 \mathrm{ab}$ & $3.73 \pm 1.67 \mathrm{a}$ & $2.00 \pm 0.41 \mathrm{~b}$ \\
Total cholesterol $(\mathrm{mg} / \mathrm{g})$ & $4.84 \pm 2.38 \mathrm{ab}$ & $6.39 \pm 2.38 \mathrm{a}$ & $2.21 \pm 3.27 \mathrm{~b}$ \\
\hline
\end{tabular}

fed high fat diets. The increased plasma triglyceride levels associated with dietary fat might be due to increased secretion of triglyceride from the liver or decreased triglyceride removal from plasma [10]. There was a report that elevated plasma TG level may be major cause of coronary artery disease because of lower levels of HDLcholesterol and increased chylomicron levels [4]. Several investigators $[22,26]$ reported that decreases in hepatic and/or plasma lipids were observed while using soy protein. Taguchi et al. [21] reported that in natto, pectin was increased $14 \%$ higher than in soybean, and that fiber such as pectin influences lipid metabolism in the intestines. We observed that Cheonggukjang supplementation decreased triglyceride and total cholesterol concentrations in serum and liver. Cheonggukjang is fermented predominantly with Bacillus species, as with natto fermentation. Several studies reported that the concentrations of fiber, vitamin $\mathrm{K}$ and isoflavonoid aglycones were increased, and new bioactive materials such as polyglutamate were created in Cheonggukjang during fermentation [7, 9]. In the present study, we conjecture that serum lipid profile and body fat accumulation were improved by complex effects of several physiology bioactive materials created during Cheonggukjang fermentation. We expect that Cheonggukjang
Fig. 1 Hepatic mRNA expression in mice. Values are means \pm SD. Values with different lowercase letters within the same panel are significantly different at $P<0.05$ by Duncan's multiple range test. $N D$ Normal diet control, $H D$ high fat diet control, HDC HD plus $40 \%$ Cheonggukjang, ACS acyl-CoA synthase, $C P T$-I carnitine palmitoyltransferase I, $A C O$ acyl-CoA oxidase, $U C P 2$ uncoupling protein 2

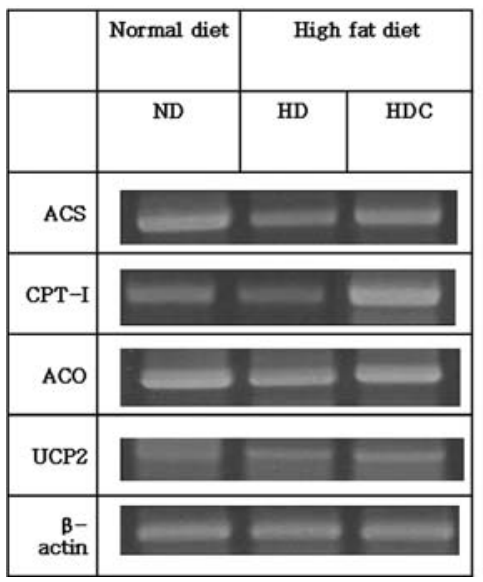

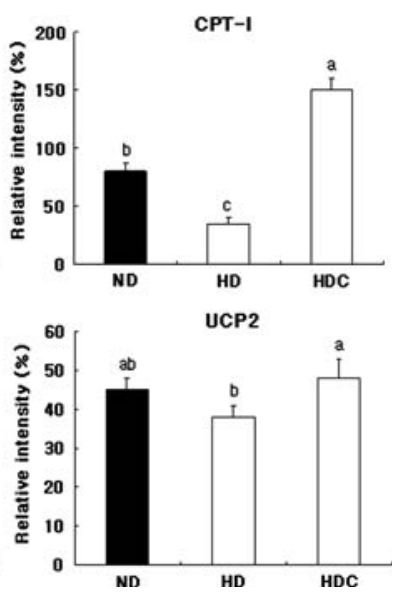


supplementation can control the occurrence of coronary heart diseases, such as arteriosclerosis, which are a consequence of poor dietary habits common in modern society, and facilitate the avoidance of diets high in fat and cholesterol.

To explore possible mechanisms for the decrease in serum and liver lipids caused by Cheonggukjang supplementation, we observed the expression levels of several genes related to lipid metabolism, energy metabolism and mitochondrial function (ACS, CPT-I, ACO and UCP2). The reaction catalyzed by ACS activates free fatty acids for use in the synthesis of phospholipids or for $\beta$-oxidation. Therefore, changes in expression levels of ACS may modulate the metabolic flux of acyl-CoA in tissue that use fatty acids for the generation or storage of energy [23]. Our results showed that hepatic ACS mRNA levels were significantly lower in the HD compared with ND groups, and tended to be higher in the HDC group than in the HD group (Fig. 1). Other studies reported that hepatic ACS mRNA levels increased or were not changed by a high fat diet [17, 20]. We expect that the differences would be caused by the differences in dietary fat content, feeding period, and kind of experimental animal.

Carnitine palmitoyltransferase-I is the rate-limiting enzyme for fatty acid $\beta$-oxidation. If the CPT-I mRNA was down-regulated in the hepatocyte, it might impair the energy substrate's transport and the energy charge required for cell regeneration. Also, it might induce dyslipidemia with accumulation of FFAs in blood and hepatocytes [6]. In a different study, liver and/or skeletal muscle CPT-I mRNA expression was increased by exercise and/or soy protein supplementation [2, 13]. Under these conditions, the expression of CPT-I is required to obtain energy from fatty acids, the primary energy substrate. In this study, hepatic CPT-I mRNA expression was increased 4.29-fold in the HDC group compared with the HD group. Therefore, we propose that increased fatty acid availability with Cheonggukjang supplementation may induce an increased expression of hepatic CPT-I mRNA (Fig. 1).

Hepatic ACO mRNA levels in the Cheonggukjang supplementation group were significantly higher than in the HD group (Fig. 1). acyl-CoA oxidase catalyzes the first step of peroxisomal fatty acid $\beta$-oxidation, and its gene expression is regulated by the peroxisome proliferatorsactivated receptors (PPARs) [24]. Peroxisome proliferators-activated receptors are ligand-activated transcriptional factors that regulate gene expression of a variety of lipid metabolizing proteins, such as ACO, enoyl-CoA hydratase/ 3-hydroxyacyl-CoA dehydrogenase, medium-chain acylCoA dehydrogenase, fatty acid binding proteins, lipoprotein lipase and apolipoproteins [18]. Therefore, PPARs are proposed to play a central role in a signaling system that controls lipid homeostasis. Although we cannot explain entirely how Cheonggukjang supplementation lowers concentration of lipids in serum and liver, one possible mechanism may be through multiple inductions of intraand extracellular fatty acid catabolism and utilization pathways (e.g., induction of fatty acid $\beta$-oxidation and TG hydrolysis), with metabolites of absorbed soy isoflavones serving as ligands that can activate PPAR.

The Cheonggukjang supplementation resulted in significantly higher UCP2 mRNA levels compared with the HD group (Fig. 1). Overall, increased mRNA of genes related to fatty acid oxidation may indicate increased fat oxidation and decreased fat accumulation in the liver. Uncoupling protein 2 is a mitochondrial inner-membrane protein that mediates proton leakage by uncoupling adenosine triphosphate synthesis [14]. Up-regulation of UCP2 mRNA by Cheonggukjang supplementation may compromise cellular adenosine triphosphate levels and decrease metabolic efficiency and, thus decrease fat accumulation.

Our results demonstrated that Cheonggukjang supplementation increased in the mRNA expression of CPT-I, a rate-limiting enzyme of fatty acid oxidation, and the mRNA expression of ACO, the first step enzyme of the $\beta$ oxidation cycle in which a fatty acyl-CoA is oxidized to 2 trans-enoyl-CoA.

In conclusion, we showed that Cheonggukjang supplementation has beneficial effects on weight gain, epididymal and back fat mass, lipids profiles of serum and liver in high fat diet fed mice. In the liver, fatty acid $\beta$-oxidation was enhanced by Cheonggukjang supplementation.

Taken together, these results suggest that Cheonggukjang supplementation improves serum lipid profiles and body fat accumulation, probably by modulating transcriptional levels of proteins and enzymes related to lipid metabolism.

Acknowledgment This research was supported by research grants from Korea Science and Engineering Foundation (KOSEF) for Biofoods Research Program, Ministry of Science and Technology (M10510120001-05N1021-00110).

\section{References}

1. Boss O, Samec S, Kuhne F, Bijlenga P, Assimacopoulos-Jeannet F, Seyboux J, Giacobino JP, Muzzin P (1998) Uncoupling protein-3 expression in rodent skeletal muscle is modulated by food intake but not by change in environmental temperature. J Biol Chem 27:5-8

2. Cha YS, Kim HY, Soh JR, Oh SH (2001) Effects of regular endurance exercise or acute-exercise and rests on levels of lipids, carnitines and carnitine palmitoyltransferase-I in rats. J Biochem Mol Biol 34:434-439

3. Choi JS, Lee JH, Young HS (1995) Anti-hyperlipidemic effect of phragmites communis and its active priniciples. J Korean Soc Food Nutr 24:523-529 
4. Goldberg IJ (1996) Lipoprotein lipase and lipolysis: central roles in lipoprotein metabolism and atherogenesis. J Lipid Res 37:693-707

5. Hill JO, Melanso EL, Wyatt HT (2000) Dietary fat intake and regulation of energy balance: implications for obesity. J Nutr 130:284S-288S

6. IJIst L, Mandel H, Oostheim W, Ruiter JP, Gutman A, Wanders RJ (1998) Molecular basis of hepatic carnitine palmitoyltransferase I deficiency. J Clin Invest 102:527-531

7. Jang CH, Lim JK, Kim JH, Park CS, Kwon DY, Kim YS, Shin DH, Kim JS (2006) Change of isoflavone content during manufacturing of Cheonggukjang, a traditional Korean fermented soyfood. Food Sci Biotechnol 15:643-646

8. Kim W, Choi K, Kim Y, Park H, Choi J, Lee Y, Oh H, Kwon I, Lee S (1996) Purification and characterization of fibrinolytic enzyme produced from Bacillus sp. Strain CK-11-4 screened from Chungkook-Jang. Appl Environ Microbiol 62:2482-2488

9. Kim SH, Yang JL, Song YS (1999) Physiological functions of Chongkukjang. Food Industry Nutrn 4:40-46

10. Lin S, Thomas TC, Storlien LH, Huang XF (2000) Development of high fat diet-induced obesity and leptin resistance in C57BI/6J mice. Int J Obes Relat Metab Disord 24:639-646

11. Miyazawa S, Hashimoto T, Yokota S (1985) Identity of longchain acyl-CoA synthetase of microsomes, mitochondria and peroxisomes in rat liver. J Biochem 98:723-733

12. Miyazawa S, Hayashi H, Hijikata M, Ishii N, Furuta S, Kagamiyama H, Osumi T, Hashimoto T (1987) Complete nucleotide sequence of cDNA and predicted amino acid sequence of rat acyl-CoA oxidase. J Biochem 262:8131-8137

13. Morifuji M, Sanbongi C, Sugiura K (2006) Dietary soya protein intake and exercise training have an additive effect on skeletal muscle fatty acid oxidation enzyme activities and mRNA levels in rats. Br J Nutr 96: 469-475

14. Nagy TR, Blaylock ML, Garvey WT (2004) Role of UCP2 and UCP3 in nutrition and obestiy. Nutrition 20:139-144

15. Nakajima N, Nozaki N, Ishihara K, Ishikawa A, Tsuji H (2005) Analysis of isoflavone content in tempeh, a fermented soybean, and preparation of a new isoflavone-enriched tempeh. J Biosci Bioeng 100:685-687
16. Recquier D, Bouillaud F (2000) The uncoupling protein homologues: UCP1, UCP2, UCP3, StUCP and AtUCP. Biochem J 345: 161-179

17. Ryu MH, Sohn HS, Heo YR, Moustaid-Moussa N, Cha YS (2005) Differential regulation of hepatic gene expression by starvation versus refeeding following a high-sucrose or high-fat diet. Nutrition 21: 543-552

18. Schoonjans K, Staels B, Auwerx J (1996) Role of the peroxisome proliferators-activated receptor(PPAR) in mediating the effects of fibrates and fatty acids on gene expression. J Lipid Res 37: 907925

19. Slavin JL, Karr SC, Hutchins AM, Lampe JW (1998) Influence of soybean processing, habitual diet and soy dose on urinary isoflavonoid excretion. Am J Clin Nutr 68: S1492-S1495

20. Suzuki H, Kawarabaysi Y, Kondo J, Abe T, Nishikawa K, Kimura S, Hashimoto T, Yamamoto T (1990) Structure and regulation of rat long-chain acyl-CoA synthetase. J Biol Chem 265:8681-8685

21. Taguchi K, Kawabata M, Ohtsuki K, Tanaka Y (1986) Changes in dietary fiber of natto and tempeh during fermentation. J Jpn Soc Nutr Food Sci 39:203-208

22. Wagner JD, Schwenke DC, Greaves KA, Zhang L, Anthony MS, Blair RM, Shadoan MK, Williams JK (2003) Soy protein with isoflavones, but not an isoflavone rich supplement, improves arterial low-density lipoprotein metabolism and atherogenesis. Arterioscler Thromb Vasc Biol 23:2241-2246

23. Weiner FR, Smith PJ, Wertheimer S, Rubin CS (1992) Regulation of gene expression by insulin and tumor necrosis factor in 3T3-L1 cells. Modulation of the transcription of the genes encoding acyl-CoA synthetase and stearoyl-CoA desaturase. J Biol Chem 266:23525-23528

24. Yang Q, Li Y (2007) Roles of PPARs on regulating myocardial energy and lipid homeostasis. J Mol Med 85:697-706

25. Yoo JY (1997) Present status of industries and research activities of Korean fermented soybean products. Microoorg Ind 23:13-30

26. Zhan S, Ho SC (2005) Meta-analysis of the effects of soy protein containing isoflavones on the lipid profile. Am J Clin Nutr 81:397-408 